\title{
Hidrocoria en semillas de Agave victoriae-reginae T. Moore, especie en peligro de extinción: Morfología y anatomía como facilitadores de la hidro-dispersión y germinación
}

\section{Hydrochory in seeds of Agave victoriae-reginae T. Moore endangered species: Morphology and anatomy as facilitators of hydro-dispersion and germination}

\author{
Jaime Sánchez-Salas ${ }^{1}$, Joel Flores ${ }^{2}$, Enrique Jurado ${ }^{3}$, Jorge Sáenz-Mata ${ }^{1}$, Pedro Orozco- \\ Figueroa ${ }^{1} \&$ Gisela Muro Pérez ${ }^{1 *}$
}

${ }^{1}$ Facultad de Ciencias Biológicas-UJED, Av. Universidad s/n. Fracc. Filadelfia, Gómez Palacio 35010, Dgo., México. ${ }^{2}$ Instituto Potosino de Investigación Científica y Tecnológica, A.C. (IPICYT), División de Ciencias Ambientales, Camino a la Presa San José No. 2055, Lomas 4a Sección, San Luis Potosí, 78210, S.L.P., México.

${ }^{3}$ Facultad de Ciencias Forestales, Universidad Autónoma de Nuevo León. A. P. 41. Carretera Nacional km 145, Linares, N.L. 67700, México

*giselamuro@ujed.mx

\begin{abstract}
RESUMEN
Agave victoriae-reginae es una especie endémica del Desierto Chihuahuense y crece sobre paredes en cañones. Posee semillas aplanadas que pueden ser dispersadas durante eventos episódicos de inundación. La presente investigación se basó en determinar posibles adaptaciones morfológicas y anatómicas en las semillas que favorecen la hidro-dispersión. Se realizó un análisis morfológico y anatómico de las semillas mediante microscopía electrónica de barrido (MEB). Se evaluaron aspectos de permeabilidad, flotabilidad, germinación y velocidad de germinación $\left(\mathrm{T}_{50}\right)$ por tratamientos y tamaños de semilla. Las semillas son diferentes en longitud, diámetro, espesor y no en peso; con forma lacriforme semiplanas y cubierta porosa con cámaras de aire en la hipodermis y son consideradas fotoblásticas neutras. Se concluye que las semillas de la especie poseen características propias para la hidro-dispersión.
\end{abstract}

Palabras clave: Flotabilidad e inundación, hidrocoria, mecanismos de dispersión.

\begin{abstract}
Agave victoriae-reginae is an endemic species from Chihuahuan Desert which grows on canyon walls. It has flattened seeds that can be dispersed during episodic flood events. This study was focused on examining possible morphological and anatomical adaptations which promote seed hydro-dispersion. A morphological and anatomical seed analysis by scanning electron microscopy (SEM) was performed. Aspects of permeability, flotation, germination and germination rate $\left(\mathrm{T}_{50}\right)$ for treatment and seed sizes were evaluated. The seeds are different in length, diameter, thickness, but not in weight; lacryform semi-flat shape and covered with porous air chambers in the hypodermis and were considered neutral photoblastic. We conclude that seeds of $A$. victoriae-reginae have hydro-dispersion characteristics.
\end{abstract}

KEYworDs: Dispersal mechanisms, flood and floating, hydrochory.

\section{INTRODUCCIÓN}

La diseminación de semillas mediante agua se denomina hidrocoria y al cuerpo dispersado diáspora hidrócora; no obstante, se considera que el agua juega un papel indirecto en la dispersión de diásporas (Granados 2002). En la década de los treinta se reportaron semillas que podían viajar sobre el agua hasta $40 \mathrm{~km}$; en años más recientes, se incluyen distancias mayores a $600 \mathrm{~km}$, como en el caso de Trifolium angustifolium L. y Plantago lagopus L. (Martínez-Orea et al. 2009). Los mecanismos que mejoran la dispersión de semillas son evidentes y fácilmente interpretables (Kerner 1898, Ridley 1930, Van der Pijl 1982); sin embargo, los que involucran rasgos dispersores de flotabilidad son 
los menos perceptibles (Willson \& Traveset 2000), como sucede en semillas de especies adaptadas al ambiente semiárido (Sánchez-Salas et al. 2012). Contrariamente, los ambientes semiáridos se caracterizan por lluvias torrenciales que provocan zonas inundables donde la mayoría de las especies poseen semillas con adaptaciones para la hidrocoria (Gutterman 2000); es decir, que son dispersadas por corrientes de agua temporales (Rheede van Oudtshoorn \& Rooyen 1999) formadas durante las lluvias (Granados 2002).

Las semillas que presentan dispersión hidrocórica son de forma navicular (Lux 1990, Bravo-Hollis \& Sánchez Mejorada 1991, Elizondo-Elizondo et al. 1994, Barthlott et al. 1997, Henrickson \& Johnston 1997, Barthlott \& Hunt 2000), hilo prominente (Bregman 1988, Lux 1990, Elizondo-Elizondo et al. 1994) y cámaras de aire con hipodermis porosa (Sánchez-Salas et al. 2012); las cuales son características propias en semillas de cactáceas. En semillas de taxones no pertenecientes a la familia Cactaceae la morfología externa es distinta; generalmente las semillas poseen forma de mejillón, mayor tamaño, hilo y micrópilo fusionados (Barthlott et al. 1997) y además crecen asociadas a vegetación riparia, como Prosopis glandulosa Torr. var. glandulosa (Villarreal et al. 2006), que posee una semilla con las características descritas anteriormente. Agave victoriae-reginae T. Moore es otra especie endémica de ambientes semiáridos, es una planta muy apreciada por coleccionistas por su valor ornamental, se encuentra enlistada en la NOM-059-SEMARNAT-2010 (DOF, 2010) como especie en peligro de extinción (Anónimo, 2010) y en el Apéndice II de CITES (Convención sobre el Comercio Internacional de Especies Amenazadas de Fauna y Flora Silvestres). Se desarrolla sobre escarpes de pendiente pronunciadas en la Sierra Madre Oriental al oeste de Nuevo León y en las serranías transversales que atraviesan Coahuila hasta el este de Durango en asociaciones vegetales de matorral xerófilo y matorral submontano. La planta es una roseta solitaria o a veces cespitosa, rara vez surculosa, acaule o con tallo corto no visible, compacta, globosa; presenta por lo regular 70 hojas, o hasta 200500 , densamente imbricadas, de color verde con bandas blancas angostas sobre las dos caras y los márgenes, rígidas o rara vez flexibles, lanceoladas o estrechamente oblongas, aquilladas o rara vez redondeadas sobre el dorso, a veces de sección casi triangular de 6-22 $\mathrm{cm}$ de largo, de $1,5-4,8 \mathrm{~cm}$ de ancho; espina terminal recta o retorcida, con frecuencia acompañada con 1 a 3 dientes cortos que coronan a los ángulos de la hoja, inflorescencia ascendente o erecta; flores en grupos de tres o a veces en pares; semillas negras, opacas o lustrosas, de forma semicircular a lacrimiformes (González-Elizondo et al. (2011). Prefiere establecerse en suelos de tipo calcáreo (Gentry 1982) por lo que se considera especie calcícola-apomórfica (Del Castillo 1996, Bravo \& Sánchez-Mejorada 1991) y puede poseer semillas con adaptaciones para la hidro-dispersión.
Bajo estas consideraciones, el presente estudio se llevó a cabo para determinar si las semillas poseen adaptaciones morfológicas y anatómicas para la dispersión por agua que favorezcan su movilización y germinación durante eventos de inundación.

\section{MATERIALES Y MÉTODOS}

Las semillas de A. victoriae-reginae fueron obtenidas en una población En Peligro de Extinción por extracción indiscriminada en Nuevo Graseros, municipio de Lerdo, Durango bajo el oficio No SGPA/DGVS/10994/14 como en González-Elizondo et al. (2011). Un lote de semillas quedó a resguardo en el banco de germoplasma en el Herbario JAAA de la FCB-UJED. Las semillas se mantuvieron a temperatura ambiente $\left(30^{\circ} \mathrm{C}\right)$ en bolsas de papel (Moreno et al. 1992; Flores et al. 2011; Sánchez-Salas et al. 2012). Posteriormente, fueron seleccionadas de acuerdo a su calidad, para lo cual se revisó la morfología externa en un estereoscopio de alta resolución (Stereo Discovery V8), descartando las semillas que presentaron anomalías. Los resultados del análisis mostraron diferencias en el tamaño de semilla, por lo que se procedió a separarlas en tres grupos: semillas pequeñas, medianas y grandes (Sánchez-Salas et al. 2006, 2015).

\section{ANÁLISIS MORFOLÓGICO}

Se utilizaron 30 semillas de cada grupo para las mediciones (longitud, diámetro, peso y espesor) con la finalidad de determinar si existen diferencias morfológicas significativas entre dichos grupos. Posteriormente, se compararon las medias a través de una prueba de Tukey (Sokal \& Rohlf 2012). El análisis incluyó una evaluación imagenológica para caracterizar las semillas a través de estereoscopio de alta resolución (Discovery V8) (Sánchez et al. 2015), siguiendo los criterios de Vázquez et al. (2011) para forma y brillo.

ANÁLISIS ANATÓMico EN SEMILLAS

El análisis se realizó mediante microscopía electrónica de barrido (MEB) de alta resolución(ESEM/QUANTA200FEI, bajo vacío / Agua) en el Instituto Potosino de Investigación Científica y Tecnológica, A. C. Se tomaron microfotografías con el ESEM en cámara de presión a 90 y $100 \mathrm{~Pa}$ con una tensión de $15,0 \mathrm{kV}$, para identificar estructuras internas que puedan influir en el proceso de hidro-dispersión (SánchezSalas et al. 2012). En el caso de los embriones no se realizó el análisis anatómico, considerando el escaso número de semillas que se tenían y se debían utilizar para las pruebas de permeabilidad, flotabilidad y germinación.

Evaluación de La PERMEABILIDAD EN SEMILlas

Para determinar si A. victoriae-reginae posee semillas 
permeables, se colocaron cinco placas Petri (unidades experimentales) a las que se les adicionaron $20 \mathrm{~mL}$ de agua de grifo, y sobre ella papel filtro. Dentro de cada unidad experimental se colocaron diez semillas (100 semillas en total), de las cuales se tenía ya el peso inicial para registrar valores reales de absorción. El registro de peso se realizó cada hora durante las primeras $8 \mathrm{~h}$. Los siguientes registros se llevaron a cabo cada ocho horas, hasta completar las $32 \mathrm{~h}$, ya que a partir de ese punto el peso se mantuvo constante (Sánchez-Salas et al. 2012). La unidad utilizada para determinar la absorción en peso fue en microgramos $(\mu \mathrm{g})$. Para calcular el porcentaje de absorción de agua se restó el peso seco inicial de las semillas al peso final, dividiendo el resultado entre el peso inicial y por último multiplicado por 100 (Dubrovsky 1998, Orozco-Segovia et al. 2007). La permeabilidad no se determinó por grupos de semillas (pequeñas, medianas y grandes), dado que la cantidad de éstas no fue suficiente, por lo que sólo se realizó el experimento para determinar que la especie posee o no semillas permeables (Sánchez-Salas et al. 2012).

\section{EVALUACIÓN DE LA FLOTABILIDAD POR TAMAÑOS DE SEMILLA}

Se preparó un lote de 50 semillas de cada uno de los tres grupos de tamaños de semilla (pequeño, mediano y grande), colocando grupos de 10 semillas en contenedores plásticos con capacidad de medio litro de agua. En el contenedor se agregaron $300 \mathrm{~mL}$ de agua y se procedió a colocar las semillas sobre la misma con una cuchara de plástico para evitar la humedad de la mano. Posteriormente, cada hora se realizó la inspección de los tratamientos, observando la cantidad de semillas que se sumergieron hasta completar 72 h (SánchezSalas et al. 2012). Los resultados fueron analizados a través de un diseño experimental completamente al azar con cinco repeticiones y 10 semillas por unidad (50 semillas por tratamiento) (Sánchez-Salas et al. 2012).

\section{ANÁLISIS DE GERMINACIÓN}

Un lote de 50 semillas por tamaño y tratamientos fueron repartidas en placas Petri (10 semillas por unidad experimental) dentro de una germinadora automática con fotoperiodo de $12 / 12 \mathrm{~h}$ luz/obscuridad y a una temperatura constante de $25^{\circ} \mathrm{C}$ (Sánchez-Salas et al. 2006). Los tratamientos fueron arena, agua y enterramiento de semillas en arena. Los cuales fueron evaluados en los tres tamaños de semillas determinados (pequeñas, medianas y grandes). El sustrato utilizado contenía arena estéril y suelo calcáreo ligeramente humedecido, ya que éste es el sustrato donde crece de manera natural la especie, aunado a las evidencias que demuestran germinación mayor al $70 \%$ empleando este sustrato (Samperio-Ruiz 2007) y agua. Los registros de germinación se evaluaron cada 24 h durante un mes (Baskin \& Baskin 1998, Sánchez-Salas et al. 2006). Para realizar la comparación de grupos con ANOVA, los valores porcentuales de germinación fueron transformados con el arcoseno de la raíz cuadrada, para normalizar los datos (Sokal \& Rohlf 2012). Una vez determinada la diferencia existente entre los tratamientos y los tamaños de semillas se realizó un análisis de varianza de una vía, seguido de la prueba de Tukey para determinar la diferencia entre las medias de los tratamientos de germinación. Finalmente, se evaluó si los tratamientos tenían efecto en el tiempo medio o velocidad de germinación (Sánchez-Salas et al. 2006, 2012, Maguire 1962), donde $M=\Sigma($ ni/t), ni: número de semillas germinadas el i-ésimo día, y t: tiempo de germinación desde la siembra hasta la germinación de la última semilla.

\section{RESULTADOS}

El análisis se dividió en una descripción morfológica externa, arreglos microestructurales de la testa y descripción anatómica de la siguiente manera:

DESCRIPCIÓN MORFOLÓGICA DE LA TESTA

1. Tamaño. Se encontraron diferencias significativas para los tres tamaños de semilla en longitud $(F=51,523$; $P<0,0001)$, diámetro $(F=38,436 ; P<0,0001)$, y espesor ( $F=22,114 ; P<0,0001)$, mientras que en peso no mostraron diferencia alguna $(F=0,971 ; P<0,383)$, como lo muestra la Tabla 1 y Figura $1 \mathrm{~b}$.

2. Forma. La semilla presenta una morfología del tipo lacrimiforme y semiplana (Vázquez et al. 2011), consiste en presencia de lados con superficies irregulares situadas en un mismo semiespacio, produciendo una cara convexa o de forma de barca (Fig. 1 b y c). Cuando la semilla no se desarrolla por completo, éstas pueden tener una forma aplanada pero hueca (García 2002).

3. Color. Las semillas poseen una testa negra brillante o lustrosa, como la reportada por Vázquez et al. 2011 y Barriada-Bernal et al. 2013; y ésta no varió en el lote de semillas revisadas (Fig. 1 b, c y d).

ArReglos microestructurales EN TESTA

1. Superficie de la testa (ST). Posee una superficie de patrón irregular rugosa causada por el relieve de la pared celular externa (Fig. 2a).

2. Contorno de las células seminales (CCS). La mayoría de las células seminales son amórficas e irregulares; sin embargo, algunas presentan un arreglo hexágono irregular limitado por un surco (Fig. 2b).

3. Relieve de las células marginales (RCM). Esta estructura da origen a los surcos y son las encargadas de la delimitación y elevaciones que presentan las células hexágono irregulares amórficas (Fig. 2c).

4. Micrópilo (M). Se localiza en la parte de mayor anchura de la semilla con posición marginal (Fig. 2a y d). 
DESCRIPCIÓN ANATÓMICA DE LA TESTA

1. Superficie de la testa (ST). La cubierta es totalmente porosa, con excepción de los relieves de las células marginales que forman los surcos. La dimensión de los poros, forma, profundidad y densidad, varían dentro de cada célula amórfica o hexágono irregular (Fig. 2b y c).

2 Hipodermis $(\mathrm{H})$. Presenta cámaras de aire distribuidas por todo el tegumento, dando una apariencia de tejido esponjoso (a causa de las cámaras de aire) que protege el embrión (Fig. 2e).

TABLa 1. Resultados del ANOVA de una vía para determinar grupos entre tamaños de semilla. Solamente el peso resultó no significativo. Promedios con error estándar y grupos que establecieron diferencia significativa entre las métricas de las semillas con un valor de $\mathrm{P}<0,05$. Letras distintas indican diferencia significativa. / One-way ANOVA results to determine groups between seed sizes. Only the weight was not significant. Averages with standard error and groups that established a significant difference between the metrics of the seeds with a value of $\mathrm{P}<0.05$. Different letters indicate significant difference.

\begin{tabular}{ccccccc}
\hline Métricas $(\mathrm{mm})$ & Pequeñas & Medianas & Grandes & $F$ & $P$ & Grupos Prueba Tukey \\
\hline Longitud & $2,986 \pm 0,136$ & $3,477 \pm 0,137$ & $4,040 \pm 0,135$ & 51,523 & 0,0001 & A, B, C \\
Diámetro & $2,847 \pm 0,169$ & $2,387 \pm 0,154$ & $1,845 \pm 0,148$ & 38,436 & 0,0001 & A, B, C \\
Peso & $0,003 \pm 0,0005$ & $0,004 \pm 0,0006$ & $0,004 \pm 0,0004$ & 0,971 & 0,383 & A, A, A \\
Espesor & $1,086 \pm 0,150$ & $0,877 \pm 0,150$ & $0,567 \pm 0,079$ & 22,114 & 0,0001 & A, B, C \\
\hline
\end{tabular}

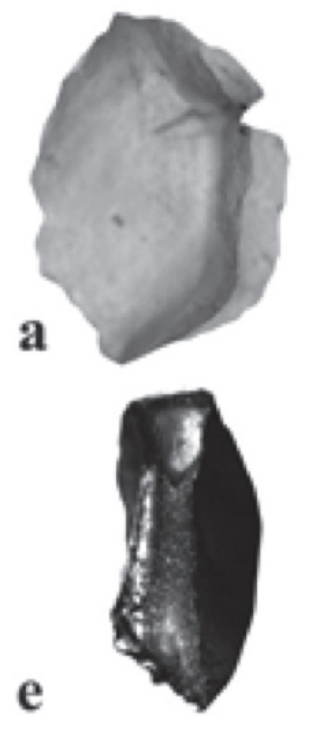

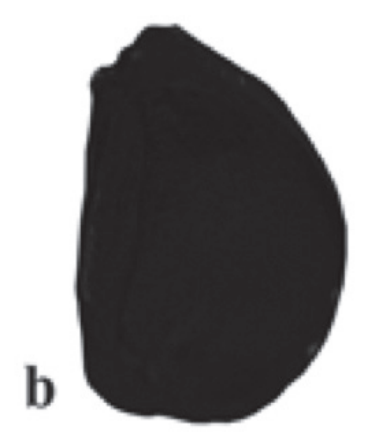

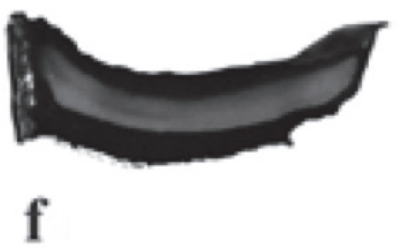

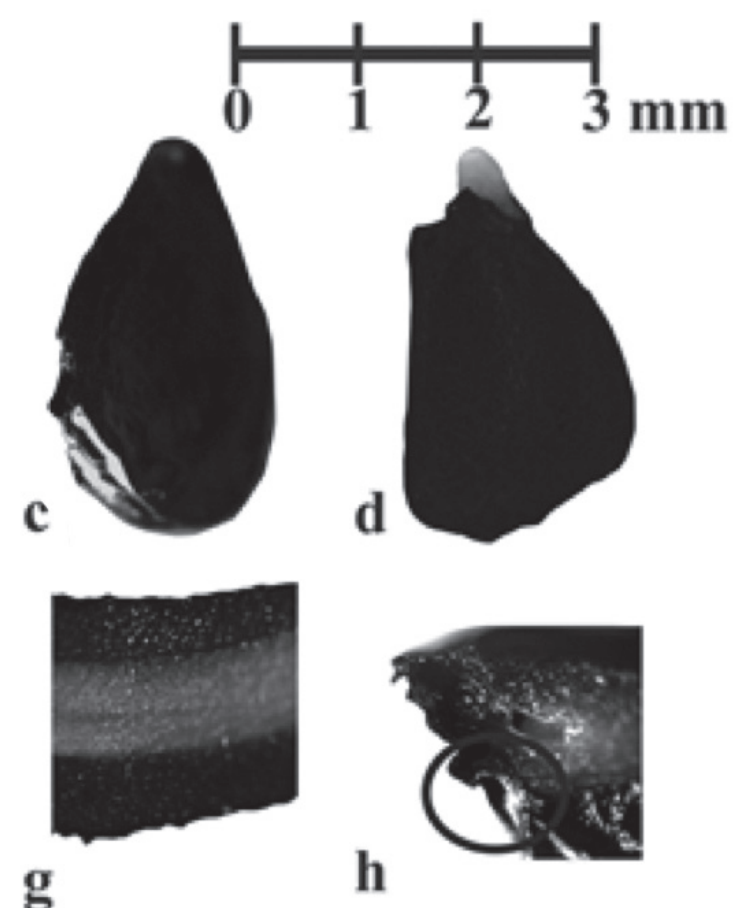

FiguRA 1. Análisis imagenológico exterior para identificación de estructuras para hidro-dispersión. Se muestra el embrión de $A$. victoriaereginae (a), forma y color de la semilla (b y c), emergencia radicular por micrópilo (d), arreglo interno de la testa y embrión (e, f y g), donde se observa una superficie porosa y posición del micrópilo resaltada en color naranja (h). Imágenes tomadas a 800 X. / External imaging analysis for identification of structures for hydro-dispersion. Embryo of A. victoriae reginae (a), shape and color of the seed (b and c), emergency root micropliar (d), internal arrangement of seed coat and embryo (e, f, and g), porous surface micropile highlighted in orange colors circle (h). Pictures taken at $800 \mathrm{X}$. 

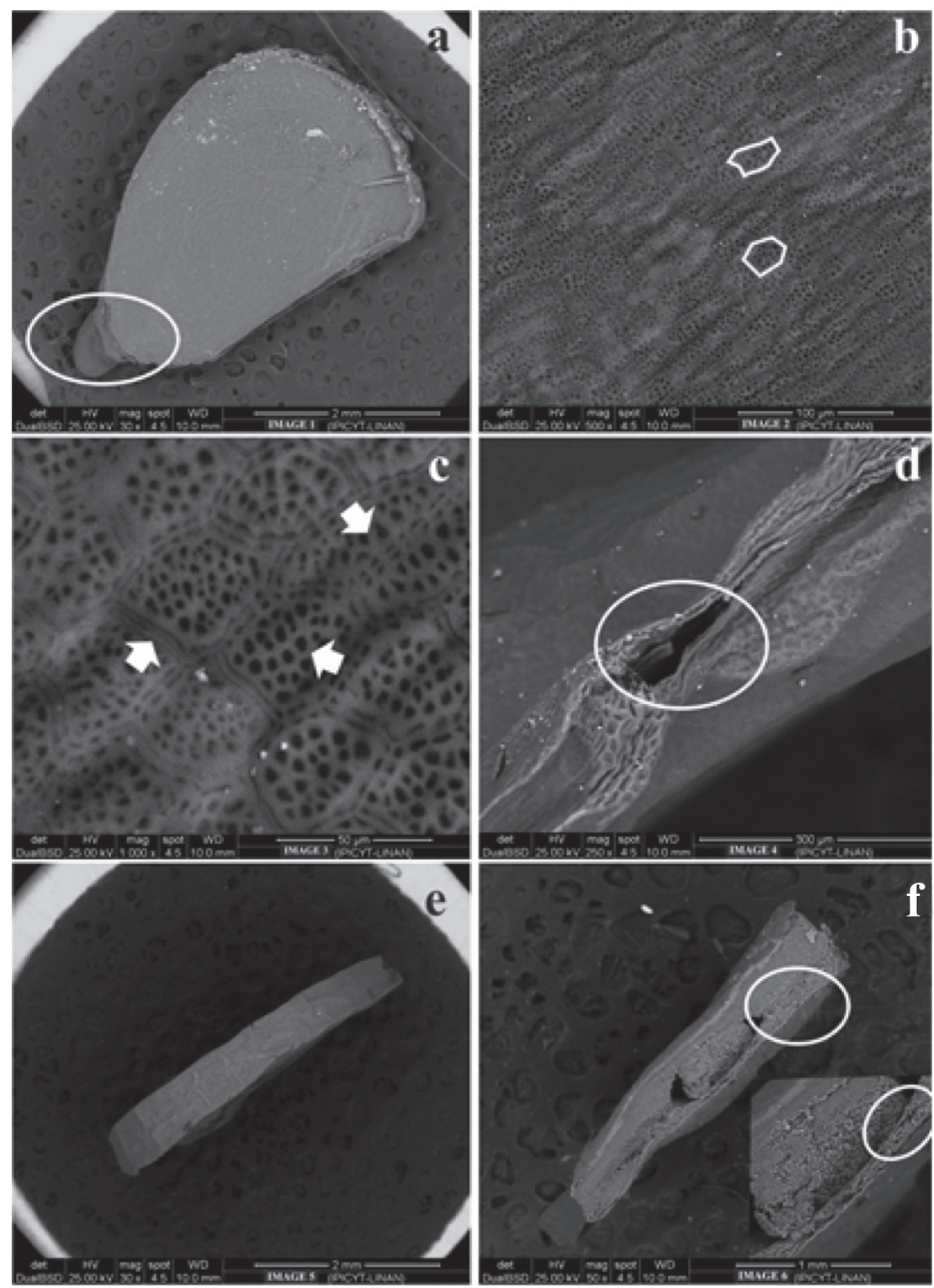

FIgURA 2. Análisis anatómico mediante microscopia electrónica de barrido (MEB) donde se muestra estructura general de la semilla (a), arreglo de la testa (b), surcos que dan origen a los límites entre células epidérmicas amórficas y hexágono irregulares (c), posición del micrópilo (d), espesor de la semilla (e) y tejido esponjoso de la hipodermis que forma las cámaras de aire (f). / Anatomical analysis using scanning electron microscopy (SEM) where general structure of the seed (a), testa arrangement (b), grooves between epidermal cells boundaries with amorphous and irregular hexagon shape (c), micropile position (d), seed thickness (e) and spongy tissue of hypodermis forming air chambers (f). 
EVALUACIÓN DE LA PERMEABILIDAD

Las semillas son consideradas permeables, por lo que están adaptadas a la absorción del agua durante eventos de inundación como los que se presentan en los sitios donde se distribuye (Tabla 2).

\section{EVALUACIÓN DE LA FLOTABILIDAD}

No se encontraron diferencias significativas respecto a la flotabilidad en las semillas de $A$. victoriae-reginae entre categorías de tamaño, esto debido a que la totalidad de las semillas flotaron durante todo el tiempo de la evaluación (Tabla 3).
EVALUACIÓN DE LA GERMINACIÓN

La germinación no se vio afectada por el tamaño de las semillas en los tratamientos de arena y agua. Sin embargo, en el tratamiento donde se colocaron las semillas enterradas, el porcentaje mostró tendencias hacia la disminución; principalmente en las semillas de tamaño grande. Esto indica que la especie posee semillas fotoblásticas positivas con cierta capacidad para germinar en ausencia de luz en menor grado (Tabla 4).

\section{VELOCIDAD DE GERMINACIÓN (MGT)}

Únicamente se encontraron diferencias significativas en semillas medianas y grandes del tratamiento semillas enterradas, pues tardaron 26 días en germinar, a diferencia de los tratamientos arena y agua, que tardaron en germinar 3 y 4 días respectivamente (Tabla 5).

TABLA 2. Resultados de ANOVA para permeabilidad. Promedios con error estándar y grupos que establecieron diferencia significativa entre grupos para permeabilidad con un valor de $P<0,05$. Letras distintas indican diferencia significativa. / One-way ANOVA results for permeability. Averages with standard error and groups that established a significant difference between for permeability with a value of $\mathrm{P}<0.05$. Different letters indicate significant difference.

\begin{tabular}{ccccc}
\hline Permeabilidad & E.E / Intervalo & F & P & Grupos Prueba Tukey \\
\hline Peso inicial $(\mu \mathrm{g})$ & $0,002 \pm 0,0002$ & 352,364 & 0,0001 & A \\
Peso final $(\mu \mathrm{g})$ & $0,006 \pm 0,0004$ & 352,364 & 0,0001 & $\mathrm{~B}$ \\
\hline
\end{tabular}

TABla 3. Resultados del ANOVA de una vía para flotabilidad a partir del tamaño de las semillas de A. victoriae-reginae. Promedios con error estándar y grupos que establecieron diferencia significativa entre las métricas de las semillas con un valor de $\mathrm{P}<0,05$. Letras distintas indican diferencia significativa. / One-way ANOVA results for buoyancy by size of $A$. victoriae-reginae seeds. Averages with standard error and groups that established a significant difference between buoyancy with a value of $\mathrm{P}<0.05$. Different letters indicate significant difference.

\begin{tabular}{ccccccc}
\hline & Pequeñas & Medianas & Grandes & $F$ & $P$ & Grupos Prueba Tukey \\
\cline { 2 - 6 } Flotabilidad & $8,800 \pm 0,3036$ & $7,800 \pm 0,6617$ & $10 \pm 0,0$ & 2,638 & 0,1123 & A, A, A \\
\hline
\end{tabular}

TABLA 4. Resultados de ANOVA para germinación, evaluando el efecto del tamaño de semillas por tratamiento. Porcentaje de germinación y error estándar con grupos que establecieron diferencia significativa con un valor de $\mathrm{P}<0,05$. Letras distintas indican diferencia significativa. / Results of ANOVA for germination for evaluation effect of the seed size by treatment. Germination percentage and standard error with groups that established significant difference with a value of $\mathrm{P}<0.05$. Different letters indicate significant difference.

\begin{tabular}{ccccccc}
\hline Germinación & Pequeñas & Medianas & Grandes & $F$ & $P$ & $\begin{array}{c}\text { Grupos Prutba } \\
\text { Tukey }\end{array}$ \\
\hline Arena & $96 \% \pm 0,0060$ & $96 \% \pm 0,0060$ & $100 \% \pm 0,00$ & 0,500 & 0,619 & $\mathrm{~A}, \mathrm{~A}, \mathrm{~A}$ \\
Agua & $94 \% \pm 0,0096$ & $100 \% \pm 0,00$ & $92 \% \pm 0,0063$ & 0,828 & 0,460 & $\mathrm{~A}, \mathrm{~A}, \mathrm{~A}$ \\
Enterradas & $74 \% \pm 0,0273$ & $64 \% \pm 0,0266$ & $42 \% \pm 0,0343$ & 1,930 & 0,088 & $\mathrm{~A}, \mathrm{~A}, \mathrm{~B}$ \\
\hline
\end{tabular}


Hidrocoria en semillas de Agave victoriae-reginae: SÁnchEZ-SALAS, J. ET AL.

TABLA 5. Resultados de ANOVA para velocidad de germinación (MGT) por tratamiento y tamaño. Velocidad de germinación (días) y error estándar con un valor de $\mathrm{P}<0,05$. Letras distintas indican diferencia significativa. / Results of ANOVA for germination speed (MGT) by treatment and size. Germination velocity and standard error with a value of $\mathrm{P}<0.05$. Different letters indicate significant difference.

\begin{tabular}{ccccccc}
\hline $\begin{array}{c}\text { Velocidad Germinación } \\
\text { en días (MGT) }\end{array}$ & Pequeñas & Medianas & Grandes & $F$ & $P$ & $\begin{array}{c}\text { Grupos Prutba } \\
\text { Tukey }\end{array}$ \\
\hline Arena & $3 \pm 0,000$ & $4 \pm 0,000$ & $4 \pm 0,000$ & 6,553 & 2,357 & A, A, A \\
Agua & $4 \pm 0,0021$ & $3,8 \pm 0,0031$ & $3,6 \pm 0,0029$ & 1,2 & 0,334 & A, A, A \\
Enterradas & $26 \pm 0,0273$ & $17,6 \pm 0,0266$ & $15,4 \pm 0,0343$ & 6,066 & 0,001 & A, B, B \\
\hline
\end{tabular}

\section{DISCUSIÓN}

\section{MoRFología DE LA SEMILlA}

Diferentes autores han propuesto que la variabilidad morfológica y tamaños de semilla influyen en la dispersión y germinación de éstas (Lux 1990, Bravo-Hollis \& SánchezMejorada 1991, Elizondo-Elizondo et al. 1994, Barthlott et al. 1997, Henrickson \& Johnston 1997, Barthlott \& Hunt 2000, Sánchez-Salas et al. 2012, 2015). Sin embargo, pocos estudios consideran que ambos atributos constituyen una presión selectiva (Farji-Brener et al. 2005) que tiende a ubicar las semillas en áreas físicas apropiadas que permiten disminuir la competencia intraespecífica. Generalmente, el tamaño de semilla se considera como una solución efectiva que favorece el proceso de germinación (Sánchez-Salas et al. 2012, 2015) y no el de dispersión y establecimiento (Ganeshaiah \& Uma Shaanker 1991). Al respecto, las semillas de Agave victoriae-reginae presentaron diferencia en longitud, diámetro y espesor, por lo que se puede considerar que tanto la dispersión como el establecimiento podrían verse favorecidos. De acuerdo con Grubb (1996), Grubb \& Coomes (1997), Grubb \& Burslem (1998) y Meyer \& Carlson (2001), el espesor de la semilla es importante en especies de ambientes áridos y semiáridos; dado que a mayor espesor, mayor tejido fotosintético que proporcionará un crecimiento acelerado principalmente en las fases juveniles (Copeland 1976, González \& Álvarez 1986), sin embargo el espesor en semillas es uno de los atributos menos estudiados.

\section{Microestructuras y ANATOMÍA DE LA TESTA}

Se ha demostrado que no sólo la forma de la semilla es determinante para generar un efecto positivo en la dispersión, sino que también las microestructuras presentes en la testa pueden contribuir a favorecer dicho proceso (Sánchez-Salas et al. 2012, 2015, Elizondo et al. 1994). Al respecto, se ha reportado que las semillas de cactáceas de semidesierto, como Astrophytum myriostigma Lemaire y A. capricorne (A. Dietr.) Britton \& Rose (Sánchez-Salas et al. 2012, 2015) y la epifita Selenicereus wittii (K. Schum) G.D. Rowley del rio Igapó del Amazonas (Barthlott et al. 1997), poseen estructuras que son llamadas cámaras de aire, consideradas como estructuras inusuales en especies de ambientes semiáridos (Chambert \& James 2009). Sin embargo, dichas estructuras son comunes en semillas de plantas de ambientes semiáridos que son dispersadas mediante hidrocoria (Barthlott et al. 1997). Estas estructuras fueron encontradas en $A$. victoriae-reginae y se encuentran alrededor de todo el tegumento formando una hipodermis porosa como las reportadas en $A$. myriostigma y $A$. capricorne (SánchezSalas et al. 2012). La testa de $A$. victoriae-reginae posee poros superficiales, los cuales no han sido reportados anteriormente en esta especie; sin embargo, semillas de Ferocactus hamatacanthus (Muehlenpf.) Britton \& Rose, Mammillaria dioica (K. Brandegee) Britton \& Rose y M. pottsii Scheer ex Engelm. las presentan en la cubierta seminal por toda la testa. De acuerdo a Barthlott \& Hunt (2000), en semillas de cactáceas los poros son formados por la escultura multicelular de la testa. Posiblemente, estas estructuras estén relacionadas con la velocidad de absorción de humedad ambiental y lluvias torrenciales, considerando que por el tipo de ecosistema en el que habita la especie le es imprescindible para la captura de agua que active el proceso germinativo.

\section{Permeabilidad}

Las semillas pueden presentar niveles elevados de permeabilidad sólo cuando poseen dos características básicas; la primera son los arreglos estructurales externos que permiten la captura y almacenamiento de la humedad ambiental (estrías o grietas), y la segunda es la cubierta tegumentaria rodeada de una gran cantidad de poros, como los reportados en semillas de Astrophytum myriostigma y A. capricorne (Sánchez-Salas et al. 2012, 2015). Las semillas de $A$. victoriae-reginae se consideran permeables, debido a que no tienen tegumentos coriosos que dificulten la absorción de agua, como los mencionados por GómezRoldan et al. (1999) y Flores \& Engelman (1976). La forma 
de la semilla ha sido reportada como factor que puede aumentar o disminuir la permeabilidad; por ejemplo, las semillas largas o globosas experimentan niveles mínimos de permeabilidad por tener menos superficie de contacto con el agua (Harper \& Benton 1966, Harper et al. 1970, Gutterman 1994, Kigel 1995), en comparación con semillas que poseen forma de canoa (Barthlott \& Hunt 2000), almeja (Barthlott et al. 1997) o lacrimosa (Vázquez et al. 2011) como la de A. victoriae-reginae, que le permite una posición boyante como las barcas y mayor contacto con el agua. Además, la alta permeabilidad que poseen las semillas evita que entren en latencia (Vásquez-Yanes \& Orozco-Segovia 1993), y por lo tanto, la germinación inicia cuando las semillas hacen contacto con el agua (Moreno et al. 2006).

\section{FLOTABILIDAD}

El concepto "pulso de inundación" (Tockner et al. 2000, Middleton 2002) asume que las condiciones hidrológicas naturales en los ríos son un requisito previo para facilitar el movimiento de diásporas durante eventos de inundación. Sin embargo, el concepto no es del todo holístico, dado que las grietas formadas por las escorrentías donde se establece $A$. victoriae-reginae no son consideradas dentro de esta concepción, pero de cierta forma son regidas por los mismos procesos hidro-ecológicos, como por ejemplo la temporalidad del agua y el tiempo de flotabilidad de las semillas. La duración de la flotabilidad es un mecanismo adaptativo que incrementa la eficiencia de la dispersión (Williamson et al. 1999, Williamson \& Costa 2000, López 2001), reportado específicamente para especies de humedales (Soons 2006). Sin embargo, se ha reportado que semillas de especies riparias (i.e. Taxodium distichum (L.) Rich, Nyssa aquatica L.) pueden flotar hasta tres meses sin perder su viabilidad (Boedeltje et al. 2004), o bien semillas de especies que son dispersadas a través de las escorrentías (i.e. Astrophytum myriostigma), que además de durar flotando por más de un mes (Sánchez-Salas et al. 2012), como se observó en las semillas de A. victoriaereginae, germinan en el agua y poseen hipocótilos flotantes que son dispersados para establecerse en sitios seguros. Se considera que la cantidad de agua generada por las variaciones pluviales provoca un efecto proporcional en el tiempo de flotación de las semillas, pues se ha determinado que aquellas que provienen de sitios inundables flotan por mayor tiempo en comparación a las de los prados (Van den Broek et al. 2005). Posiblemente, por esta razón las semillas de $A$. victoriae-reginae flotan por más de un mes, ya que son dispersadas a través de escorrentías.

\section{GerminaCión}

El efecto de tamaño de semillas ha sido reportado como factor que influye en la capacidad y velocidad de la germinación (Sánchez-Salas et al. 2012, 2006), tanto entre especies (Bowers \& Pierson 2001) como dentro de la misma especie (Ayala-Cordero et al. 2004). Sin embargo, en $A$. victoriae-reginae el efecto del tamaño y los tratamientos no favorecieron o afectaron el porcentaje de germinación, aun y cuando una diferencia mínima en tamaño o peso puede alterar la capacidad germinativa (Baloch et al. 2001), esperando generalmente un comportamiento donde las semillas grandes germinan en mayor porcentaje e incluso el doble (Tenorio-Galindo et al. 2008). De acuerdo con Moegenburg (1996), el tamaño de la semilla se relaciona directamente con el tamaño de la planta, por lo que las plántulas más grandes provienen de semillas grandes que poseen mayores probabilidades de germinar y sobrevivir (Tenorio-Galindo et al. 2008, Leishman et al. 2000), como en Helianthus annuus L. (Krishnaveni \& Sivasubramanian 2001). Hendrix (1984) determinó que las semillas grandes poseen mayor porcentaje de germinación, y en este sentido, los resultados obtenidos en este estudio no concuerdan del todo con el autor, pues el porcentaje de germinación se inclinó mayormente en las semillas pequeñas en dos de los tres tratamientos evaluados. Posiblemente esto se debe a que a mayor tamaño, mayor tiempo en germinar, como lo consideran Gomes et al. (2001), Tungate et al. (2002), Kikuzawa \& Koyama (1999), Teketay \& Granstrom (1997), Milberg et al. (1996) y Harper et al. (1970).

Además de lo considerado por los autores anteriormente citados, es necesario tomar en cuenta que el tegumento de la semilla está constituido por tejido esponjoso que forma las cámaras de aire (Sánchez-Salas et al. 2012) y por consecuencia, a menor tamaño de semilla, menor número de cámaras de aire, que inducirán la germinación en menor tiempo.

\section{VELOCIDAD DE GERMINACIÓN (MGT)}

Se considera que la temperatura es el factor principal que regula la velocidad de germinación a través de las fluctuaciones estacionales y cotidianas (Taylor et al. 1999) del microhábitat. Sin embargo, parece que el factor determinante que afecta la germinación en $A$. victoriaereginae es la luz, tanto en semillas pequeñas como en semillas enterradas. En este sentido, se considera que la especie posee semillas insensibles a la luz u oscuridad (neutras), pues germinan de manera indistinta en ambas condiciones (Takaki 2001). Los resultados son similares a los reportados por Enríquez Peña et al. (2004), donde no existe un patrón claro de germinación en presencia o ausencia de luz para semillas de Taxodium mucronatum, pues éstas son tolerantes a la sombra al germinar también bajo condiciones de oscuridad como las enterradas de $A$. victoriae-reginae.

\section{CONCLUSIONES}

Las semillas de $A$. victoriae-reginae presentan adaptaciones 
morfológicas y anatómicas para la hidrodispersión, como la forma lacriforme semiplana, que le proporciona flotabilidad, una cubierta porosa con hipodermis rodeada de cámaras de aire, que facilitan la hidrodispersión, y semillas permeables, que pueden capturar agua en periodos cortos de inundación y que favorecen la germinación postlluvias. Además, las semillas tienen la capacidad de germinar en ausencia de luz en menor tiempo, favoreciendo la germinación de semillas medianas y grandes, particularmente cuando las semillas caen entre grietas durante el proceso de hidro-dispersión durante las lluvias. Esto explica el establecimiento de plantas adultas entre grietas, las cuales se denominan rupícola-casmófitas.

\section{AGRADECIMIENTOS}

A Iris Peña Maldonado de la División de Materiales Avanzados del Instituto Potosino de Investigación Científica y Tecnológica, A.C. por las imágenes proporcionadas mediante microscopia electrónica de barrido (MEB).

\section{REFERENCIAS}

Ayala-Cordero, G., Terrazas, T., López-Mata, L., Trejo, C. 2004. Variación en el tamaño y peso de la semilla y su relación con la germinación en una población de Stenocereus beneckei. Interciencia 29(12): 692-697.

Baloch, H.A., DiTomasso, A., Watson, A.K. 2001. Intrapopulation variation in Abutilon theophrasti seed mass and its relationship to seed germinability. Seed Science Research 11(4): 335-343.

Barriada-Bernal, G., Almaraz-Abarca, N., GallardoVelázquez, T., Torres-Morán, I., Herrera-Arrieta, Y., González-Elizondo, S., Delgado-Alvarado, E. 2013. Seed vigor variation of Agave durangensis Gentry (Agavaceae). American Journal of Plant Sciences 4(11): 2227-2239.

Barthlott, W., Hunt, D. 2000. Seed-diversity in the Cactaceae subfam. Cactoideae. Succulent Plant Research 5: 1-173.

Barthlott, W., Porembski, S., Kluge, M., Hopke, J., Schmidt, L. 1997. Selenicereus wittii (Cactaceae): An epiphyte adapted to Amazonian Igápo inundation forests. Plant Systematics and Evolution 206(1): 175-185.

Baskin, C.C., Baskin, J.M. 1998. Seeds. Ecology, biogeography, and evolution of dormancy and germination. Academic Press, San Diego. 666 pp.

Boedeltje, G., Bakker, P.J., Ten-Brinke, A., Van Groenendael, M.J., Soesbergen, M. 2004. Dispersal phenology of hydrochorous plants in relation to discharge, seed release time and buoyancy of seeds: The flood pulse concept supported. Journal of Ecology 92: 786-796.

Bowers, J.E., Pierson, E.A. 2001. Implications of seed size for seedling survival in Carnegiea gigantea and Ferocactus wislizeni (Cactaceae). Southwestern Naturalist 46(3): 272281.
Bravo-Hollis, H., SÁnchez-Mejorada, H.R. 1991. Las Cactáceas de México, vol. II. Universidad Nacional Autónoma de México, México, D.F. 404 pp.

Bregman, R. 1988. Forms of seed dispersal in Cactaceae. Acta Botanica Neerlandic 37(3): 395-402.

Chambert, S., James, C.S. 2009. Sorting of seeds by hydrochory. River Research and Applications 25(1): 48-61.

Copeland, L.O. 1976. Principles of Seed Science and Technology. Burgess Pub. Comp., Minnaeapolis, Minnesota, pp. 164165 .

Copeland, L.O., McDonald, M.B. 2001. Principles of seed science and technology. 4th ed. Norwell, Massachusetts: Kluwer Academic Publishers. 488 pp.

Del Castillo, R.F. 1996. Ensayo sobre el fenómeno calcícolacalcífuga en cactáceas mexicanas. Cactáceas y Suculentas Mexicanas. Tomo XLI (1): 3-11.

Diario Oficial de la Federación (DOF). 2010. NORMA OFICIAL MEXICANA NOM-059-SEMARNAT-2010. Protección ambiental-especies nativas de México de flora y fauna silvestres-categorías de riesgo y especificaciones para su inclusión, exclusión o cambio - Lista de especies en riesgo. Secretaría de Medio Ambiente y Recursos Naturales. Diario Oficial de la Federación. México, D.F. 40 pp.

Dubrovsky, J.G. 1998. Discontinuous hydration as a facultative requirement for seed germination in two cactus species of the Sonoran Desert. Journal of the Torrey Botanical Society 125(1): 33-39.

Elizondo-Elizondo, J., Valdés-Reyna, J., Arias-Montes, S.A., Hatch, L.S. 1994. Micromorfología de las semillas de algunas cactáceas de la tribu Cacteae (Cactaceae). Cactáceas y Suculentas Mexicanas 39(3): 59-67.

Enríquez Peña, G.E., Suzán Azpiri, H., Malda Barrera, G. 2004. Viabilidad y germinación de semillas de Taxodium mucronatum (Ten.) en el estado de Querétaro, México. Agrociencia 38(3): 375-381.

Farji-Brener, A.G., Durán, S., Valerio, A., Herbas, E., Castañeda, M., Ochoa, J., Romo, M. 2005. La semilla de Campsiandra angustifolia (Fabaceae:Caesalpiniodeae) como un reflejo de las presiones selectivas sobre su dispersión y establecimiento. Revista de Biología Tropical 53(1-2): 63-71.

Flores, J., Jurado, E., Chapa-Vargas, L., Ceroni-Stuva, A., Dávila-Aranda, P., Galíndez, G., Gurvich de León-Lobos, P., Ordóñez, C., Ortega-Baes, P., Ramírez, P., Sandoval, A., Seal, C.E., Ullian, T., Pritchard, H.W. 2011. Seeds photoblastism and its relationship with some plant traits in 136 cacti species. Environmental and Experimental Botany 71: 79-88.

Flores, E.M., Engleman, E.M. 1976. Apuntes sobre anatomía y morfología de las semillas de cactáceas. II. Caracteres de valor taxonómico. Revista Biología Tropical 24: 299-321.

Ganeshaiah, K.N., Uma ShaAnKer, R. 1991. Seed size optimization in a wind dispersed tree Butea monosperma: A tradeoff between seedling establishment and pod dispersal efficiency. Oikos 60(1): 3-6.

García-MendozA, A. 2002. Distribution of the genus Agave (Agavaceae) and its endemic species in Mexico. Cactus y Suculentas Society of America 74: 177-187.

Gentry, H.S. 1982. Agaves of Continental North America. The University of Arizona Press, Tucson, Arizona. 670 pp. 
Gomes, V., Madeira, J.A., Wilson Fernández, J., Lemos Filho, J.P. 2001. Seed dormancy and germination of sympatric species of Chamaecrista (Leguminosae) in a rupestrian field. International Journal of Ecology and Environmental Sciences 27: 191-197.

Gómez-Roldán, M.C., Pérez-Herrera, P., Valdéz-Carrasco, J., Acosta-Gallegos, J.A. 1999. Characteristics of the seed coat in wild and cultivated Phaseolus vulgaris L. Annual Report of the Bean Improvement Cooperative 42(2): 9192.

González, S.S., Álvarez, M.G. 1986. Efecto de la imbibición en la germinación de cuatro especies frutales. Tesis de Licenciatura. Universidad Nacional Autónoma de México. México, D.F. 121 pp.

GonzÁlez-Elizondo, M.S., GonzÁlez-Elizondo, M., LóPez, I.L., Reséndiz-Rojas, L., Tena-Flores, J.A., RetanaRentería, F.I. 2011. El complejo Agave victoriae-reginae (Agavaceae). Acta Botánica Mexicana 95: 65-94.

Granados, S.D. 2002. Ecología y dispersión de las plantas. Universidad Autónoma Chapingo. Estado de México, 115 pp.

GruBB, P.J. 1996. Rainforest dynamics the need for new paradigms. In: Edwards, D.S., Booth, W.E., Choy, S.C. (eds.). Tropical Rainforest Research, 215-233. Klawer Academic Publisher, UK.

Grubb, P.J., Coomes, D.A. 1997. Seed mass and nutrient content in nutrient-starved tropical rainforest in Venezuela. Seed Science Research 7(3): 269-280.

GrubB, P.J., Burslem, D.F.R.P. 1998. Mineral nutrient concentrations as a function of seed size within seed crop: Implications for competition among seedlings and defense against herbivory. Journal of Tropical Ecology 14(2): 177185.

GutTerman, Y. 1994. Strategies of seed dispersal and germination in plants inhabiting deserts. Botanical Review 60(4): $373-$ 425.

GutTERman, Y. 2000. Environmental factors and survival strategies of annual plant species in the Negev Desert, Israel. Plant Species Biology 15(2): 113-125.

Harper, J.L., Benton, R.A. 1966. The behavior of seeds in soil: II. The germination of seeds on the surface of a water supplying substrate. Journal of Ecology 54(1): 151-166.

Harper, J.L., Lovell, P., Moore, K.G. 1970. The shapes and sizes of seeds. Annual Review of Ecology and Systematics 1: 327-356.

HENDRIX, S.D. 1984. Variation in seed weight and its effect on germination in Pastinaca sativa L. (Umbelliferae). American Journal of Botany 71(6): 795-802.

Henrickson, J., Johnston, M.C. 1997. A flora of the Chihuahuan Desert Region. Edition 1. 2. Vol. 1. Manuscript. 280 pp.

Kerner, A. 1898. The Natural History of Plants: Their Form, Growth, Reproduction and Distribution (English translation by F.W. Oliver), 2 vols. Holt, New York. 256 pp.

Kigel, J. 1995. Seed germination in arid and semiarid regions. In: Kigel, J., Galili, G. (eds.), Seed development and germination, 645-699. Marcel Dekker Inc, New York, USA.

Kikuzawa, K., Koyama, H. 1999. Scaling of soil water absorption by seeds: an experiment using seed analogues. Seed Science Research 9(2): 171-178.
Krishnaveni, K., Sivasubramanian, K. 2001. Effect of seed size on seed quality in sunflower cv. Morden. Madras Agricultural Journal 88: 133-134.

Leishman, M.R., Wright, I.J., Moles, A.T., Westoby, M. 2000. The evolutionary ecology of seed size. In: Fenner, M. (ed.). Seeds: The ecology of regeneration in plant communities, 31-57. CABI International, Wallingford, UK.

LÓPEZ, O.R. 2001. Seed flotation and post flooding germination in tropical terra firmer and seasonally flooded forest species. Functional Ecology 15(6): 763-771.

Lux, A. 1990. Ultrastructure of seeds in the family Cactaceae. Mature embryo of Astrophytum asterias (Zucc.) Lem. Acta Facultatis Rerum Naturalium Universitatis Comenianae. Physiologia Plantarum 26: 59-66.

MaguiRe, J.D. 1962. Speed of germination-aid in selection and evaluation for seedling emergence and vigour. Crop Science Madison 2(2): 176-177.

Martínez-Orea, Y., Castillo, A.S., Guadarrama, C.P. 2009. La dispersión de frutos y semillas. Facultad de Ciencias. Del Herbario. Revista CIENCIAS-UNAM. 38-41.

Meyer, S.E., Carlson, S.L. 2001. Achene mass variation in Ericameria nauseosus (Asteraceae) in relation to dispersal ability and seedling fitness. Functional Ecology 15(2): 274-281.

Middleton, B.A. 2002. Flood Pulsing and Wetlands: Restoring the Natural Balance. Beth Middleton (ed.), New York. 320 pp.

Milberg, P., Anderson, L., Elfverson, C., Regnér, S. 1996. Germination characteristics of seeds differing in mass. Seed Science Research 6(4): 191-197.

Moegenburg, S.M. 1996. Sabal palmetto seed size: causes of variation, choices of predators and consequences for seedlings. Oecologia 106(4): 539-543.

Moreno, F., Guido, A.P., Stanislav, V.M. 2006. Efecto de la testa sobre la germinación de semillas de caucho (Hevea brasiliensis Muell.). Agronomía Colombiana 24(2): 290295.

Moreno, N., López, J., Arce, L. 1992. Aspectos sobre las semillas y su germinación de Echinomastus mariposensis Hester. Cactáceas y Suculentas Mexicanas 37: 21-27.

Muro-Pérez, G., Jurado, E., Flores, J., Sánchez, J. 2013. Efecto de la densidad de semillas en la germinación de tres especies del género Astrophytum (Cactaceae). Gayana Botánica 70(1): 26-30.

Orozco-Segovia, A., Márquez-Guzmán, J., Sánchez-Coronado, M.E., Gamboa de Buen, A., Baskin, J.M., Baskin, C.C. 2007. Seed anatomy and water uptake in relation to seed dormancy in Opuntia tomentosa (Cactaceae Opuntioideae). Annals of Botany 99(4): 581-592.

Rheede van Oudtshoorn, K., Rooyen, M.W. 1999. Dispersal Biology of Desert Plants: Adaptations of Desert Organisms. Springer, London. 244 pp.

RidLEY, H.N. 1930. The dispersal of plants throughout the world. Ashford, UK: Reeve \& Co. New Phytologist 94: 313-317.

SAmperio-Ruíz, O.D. 2007. Evaluación de la efectividad de 11 tratamientos para la germinación de la cactácea amenazada Astrophytum capricorne (A. Dietr.) Britton \& Rose (Cactaceae), de acuerdo al tamaño de semilla y tipo de sustrato. Tesis de licenciatura. Facultad de Ciencias Biológicas. Universidad Juárez del Estado de Durango. 105 pp. 
Hidrocoria en semillas de Agave victoriae-reginae: SÁnchez-SALAS, J. ET AL.

SÁnchez, B. 1983. Factores que influyen en la fisiología de germinación de la Urtica urens L. Tesis de Licenciatura. Facultad de Agronomía, Universidad Nacional de Colombia, Bogotá. 175 pp.

Sánchez-Salas, J., Flores, J., Martínez-García, E. 2006. Efecto del tamaño de la semilla en la germinación de Astrophytum myrostigma Lemaire. (Cactaceae), especie amenazada de extinción. Interciencia 31(5): 371-375.

Sánchez-Salas, J., Flores, J., Muro, G., Arias-Montes, S., JuRADO, E. 2015. Morfometría de semillas en la cactácea amenazada de extinción Astrophytum myriostigma Lemaire. Polibotánica 39: 119-131.

Sánchez-Salas, J., Jurado, E., Flores, J., Estrada-Castillón, E., Muro-PÉrez, J. 2012. Desert species adapted for dispersal and germination during floods. Experimental evidence in two Astrophytum species (Cactaceae). Flora 207(10): 707711.

SoKal, R.R., Rohlf, F.J. 2012. Biometry: The principles and practice of statistics in biological research. W.H. Freeman and Company, New York. 937 pp.

Soons, M.B. 2006. Wind dispersal in freshwater wetlands: Knowledge for conservation and restoration. Applied Vegetation Science 9(2): 271-278.

Soriano, J.P., Naranjo, M.E., Rengifo, C., Figuera, M., Rondón, M., RuIz, L.R. 1999. Avian consumers of columnar cacti fruits in the semiarid enclave of Lagunillas, Merida, Venezuela. Ecotropicos 12(2): 91-100.

TAKAKI, M. 2001. New proposal of classification of seeds based on forms of phytochrome instead of photoblastism. Brazilian Journal of Plant Physiology, Brasília 13(1): 104-108.

TAylor, J.P., Wester, D.B., Smith, L.M. 1999. Soil disturbance, flood management, and riparian woody plant establishment in the Rio Grande floodplain. Wetlands 19(2): 372-382.

Teketay, D., Granström, A. 1997. Germination ecology of forest species from the highlands of Ethiopia. Journal of Tropical Ecology 13(6): 793-803.

Tenorio-Galindo, G., Rodríguez-Tejo, A.D., López-Ríos, G. 2008. Efecto del tamaño y color de la semilla en la germinación de
Cecropia obtusofolia Bertol (Cecropiaceae). Agrociencia 42(5): 585-593.

Tockner, K., Malard, F., Ward, J.V. 2000. An extension of the flood pulse concept. Hydrological Processes 14(16-17): 2861-2883.

Tungate, K.D., Susko, D.J., Rufty, T.W. 2002. Reproduction and offspring competitiveness of Senna obtusifolia are influenced by nutrient availability. New Phytologist 154(3): 661-669.

Van den Broek, T., Van Diggelen, R., Bobbink, R. 2005. Variation in seed buoyancy of species in wetland ecosystems with different flooding dynamics. Journal Vegetation Science 16(5): 579-586.

VAN DER PIJL, L. 1982. Principles of Dispersal in Higher Plants. Springer-Verlag, Berlin. 154 pp.

Vázquez Días, E., García Nava, R., Peña Valdivia, C.B., Ramírez Tobías, H.M., Morales Ramos, V. 2011. Tamaño de la semilla, emergencia, y desarrollo de la plántula de maguey (Agave salmiana Otto ex Salm-Dyck). Revista Fitotecnia Mexicana 34(3): 167-173.

Vázquez-Yanes, C., Orozco-Segovia, A. 1993. Patterns of seed longevity and germination in the tropical rainforest. Annual Review of Ecology and Systematics 24: 69-87.

Villarreal Quintanilla, J.Á., Carranza P., M.Á., Estrada Castillón, E., Rodríguez G., A. 2006. Flora riparia de los Ríos Sabinas y San Rodrigo, Coahuila, México. Acta Botánica Mexicana 75: 1-20.

Williamson, G. B., Costa, F. 2000. Dispersal of Amazonian Trees: Hydrochory in Pentaclethra macroloda. Biotropica, 32: 548-552

Williamson, G.B., Costa, F., Minte Vera, C.V. 1999. Dispersal of Amazonian tress: hydrocory in Swartzia polyphylla. Biotropica 31: 460-465.

Willson, F.M., Traveset, A. 2000. The Ecology of seed dispersal. In: Fenner, M. (ed.). The Ecology of Regeneration in Plant Communities, 85-110. CABI International, Wallingford, UK.

Recibido: 24.11.2016

Aceptado: 25.09.2017 